\author{
A novel method for Measuring Mass by Image Processing \\ Xiaomin Tan ${ }^{1, a}$, Jintian Lei ${ }^{1, b}$, Hongmei Bi ${ }^{1}$, Tianpeng Liu $^{1}$, Xuejun Zhang ${ }^{1,2, c}$ \\ and Ximing Wang ${ }^{2}$ \\ ${ }^{1}$ School of Computer and Electronic Information, Guangxi University, Nanning, Guangxi 530004, P. \\ R. China \\ ${ }^{2}$ IPILab, Biomedical Engineering Dept, University of Southern California, Los Angeles, CA 90033, \\ USA. \\ aashleytan22@hotmail.com, ${ }^{b}$ gxuljt@163.com, xjzhang@gxu.edu.cn
}

Keywords: Thin Plate Spline (TPS) Fast Fourier Transform (FFT) Mass Spectrum Bending Energy Computer Vision

\begin{abstract}
Image processing in terms of grid deformation was applied to measure the mass of objects. In spatial domain, Thin Plate Spline (TPS) method was employed to provide the value of minimum bending energy produced during grid deformation, and quantized mass of object; in frequency domain, Fast Fourier Transform (FFT) algorithm was to calculate the changed value of spectrum both before and after deformation in a power spectrum region. After drawing the relation curve between spatial/frequency feature and corresponding mass, mass of unknown objects can be measured on images before and after deformation. Results showed that, as the acting force that objects withstood became larger, values of bending energy increased, effects for spectrum spreading became more obvious as well. Objects having good flexibilities were compared and analyzed through an image experiment and it showed that tensile belt fitted the experiment pretty well. Different values of acting force were applied to the belt and its deformation effect was remarkable. The result was linear distribution and in accordance with the theoretical expectation.
\end{abstract}

\title{
Introduction
}

For human being, more than 80 percents of information comes from vision and other information is obtained through tough feeling, auditory sense and gustation. More information will be produced if vision and touch feeling are combined together, but it will increase the complexity in a robot system as more sensors are required. Robot vision covers the shortage of sensors (touch feeling) thanks to the rapid development of image processing.

Measurement and analysis for object mass or its hardness using image processing are uncommon so far. Some studies were reported about the measurement of the stiffness of the internal organs such as heart ${ }^{[1]}$ or liver ${ }^{[2]}$ used MR tagging image. In our study, a method differs from usual hardware solution was employed to measure the mass of objects that was hanged with a soft belt having good flexibility, such as tensile belt. The measurement is undergone on a pair images with grids on belt before and after the placement of weight sets. This study is to investigate the possibility of measuring mass by quantifying the grid deformation.

\section{Experimental material}

Grid dots are drawn in the center area of tensile belt with red color as showed in Fig.1(a). Tensile belt is fixed horizontally and the dotting rectangle known as "drawing points" coincides with the region appearing deformation easily. A series of weights with different values hang under the drawing points, resulting different deformation degree of tensile belt, which showed in Fig.1(b)-Fig.1(d). 


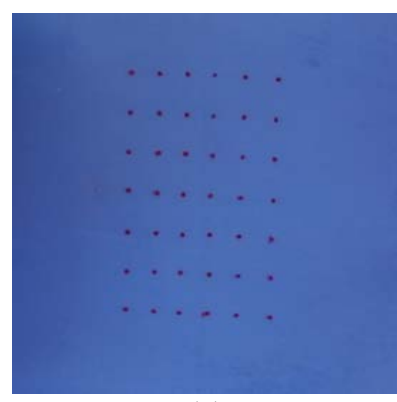

(a)

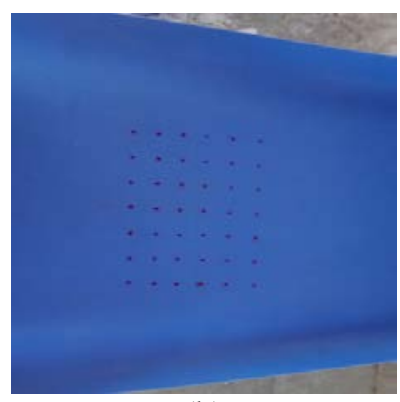

(b)

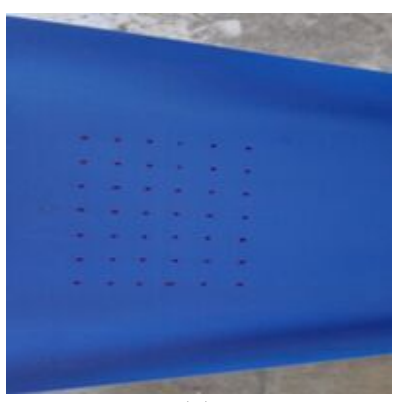

(c)

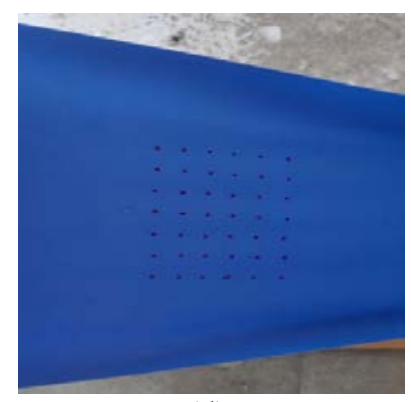

(d)

Fig. 1 Dotting rectangle(a) and the deformed grids with 550g(b),1200g(c),2000g(d).

\section{TPS Method and bending energy (in spatial domain)}

Thin Plate Spline (TPS) ${ }^{[3]}$ is often used in spatial deformation of an image. It is a mapping from control points to the correspondent points in floating image. TPS is actually a interpolation with multi variables ${ }^{[4]}$. In 2-dimision spatial, Energy function is:

$$
I_{f}=\iint_{R^{2}}\left(\left(\frac{\partial^{2} f}{\partial x^{2}}\right)^{2}+2\left(\frac{\partial^{2} f}{\partial x \partial y}\right)^{2}+\left(\frac{\partial^{2} f}{\partial y^{2}}\right)^{2}\right) d x d y
$$

The minimum bending energy value of $I_{f}$ can be calculated by:

$$
I_{f}=V\left(L_{n}{ }^{-1} K L_{n}{ }^{-1}\right) V^{T} \text {. }
$$

where

$$
P=\left[\begin{array}{ccc}
1 & x_{1} & y_{1} \\
1 & x_{2} & y_{2} \\
\ldots & \ldots & \ldots \\
1 & x_{n} & y_{n}
\end{array}\right], V=\left[\begin{array}{llll}
x_{1}^{\prime} & x_{2}^{\prime} & \ldots & x_{n}^{\prime} \\
y_{1}^{\prime} & y_{2}^{\prime} & \ldots & y_{n}^{\prime}
\end{array}\right], K=\left[\begin{array}{cccc}
0 & U\left(r_{12}\right) & \ldots & U\left(r_{1 n}\right) \\
U\left(r_{21}\right) & 0 & \ldots & U\left(r_{2 n}\right) \\
\ldots & \ldots & \ldots & \ldots \\
U\left(r_{n 1}\right) & U\left(r_{n 2}\right) & \ldots & 0
\end{array}\right]
$$

and

$$
L=\left[\begin{array}{l|l}
K & P \\
\hline P^{T} & O
\end{array}\right] .
$$

A target landmark was chosen in the dotting rectangle and two sets of coordinate values $\left(\mathrm{P}\right.$ and $\left.\mathrm{P}^{\prime}\right)$ are obtained. $\mathrm{P}$ is the coordinate value without hanging weights while $\mathrm{P}^{\prime}$ is with weights. These values are imported in the TPS program as a pair landmark to calculate minimum bending energy. Flow chart of TPS processing is shown in Figure 2.

Image $\rightarrow$ Mark P and P' manually $\rightarrow$ Calculate TPS minimum bending $\rightarrow$ Spatial features

Fig.2 Flow chart of TPS method

\section{FFT method (in frequency domain)}

Fourier transform is an important tool in image processing that enables to obtain and analyze image information in frequency domain. Fourier transform is very time consuming as Discrete Fourier transform (DFT) is applied to 2-dimension digital image in frequency domain. In order to reduce the calculation time, Fast Fourier transform $(\text { FFT })^{[5]}$ is proposed as a fast vision of DFT, and can be expressed by the formula:

$$
F(u)=\frac{1}{2 M} \sum_{x=0}^{2 M-1} f(x) W_{2 M}^{u x}
$$




$$
=\frac{1}{2}\left\{\frac{1}{M} \sum_{x=0}^{M-1} f(2 x) W_{2 M}^{u(2 x)}+\frac{1}{M} \sum_{x=0}^{M-1} f(2 x+1) W_{2 M}^{u(2 x+1)}\right\} .
$$

where $\mathrm{u}=0,1,2, \ldots, \mathrm{M}-1$.

In order to make a clean background, the red dots on the belt are firstly extracted. Then the image is compressed to $256^{*} 256$ for frequency processing. Finally, FFT is applied to the compressed image to calculate the spectrum values. The flowchart of FFT processing is shown in Figure 3.

To extract the red dots from blue background, color image without deformation in Fig. 1a is transferred into gray value image by selecting the pixels with red color. Red dots are chosen on the tensile belt and only their pixel values is greater than a threshold are remained in a pre-processed image, from which FFT is used to make a power spectrum image in Figure 4. Red dots in all captured images are extracted using the same threshold.

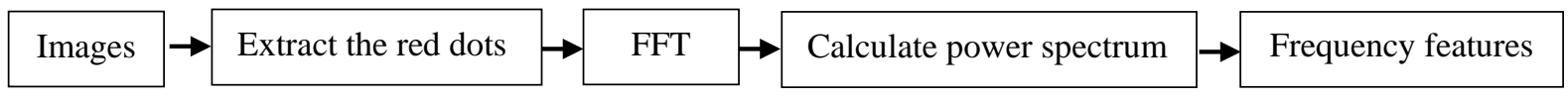

Fig.3 Flow chart of FFT method

There is only a cross in Figure 4(a) and(c), which indicate the background color and belt edge may impact the result of spectrum values. Figure 4(b) and (d) show the spectrum images become more clearly, reflecting that more information of deformation are presented in the spectrum image.

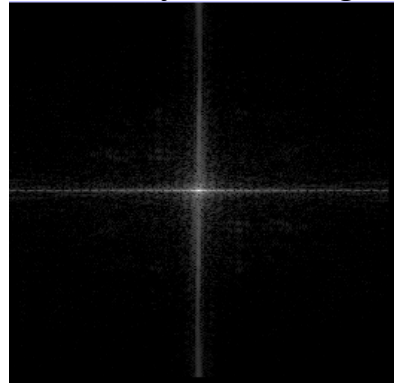

(a)

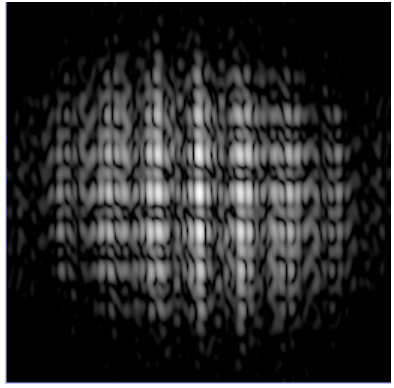

(b)

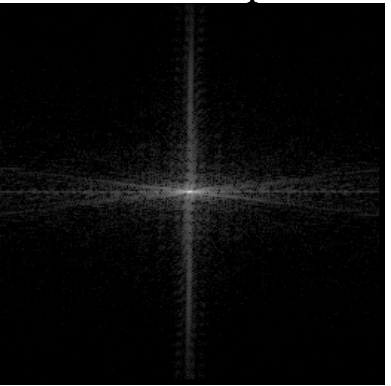

(C)

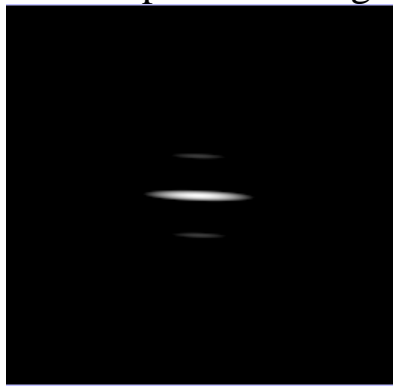

(d)

Fig.4 Spectrum images with background color and without weight (a); Spectrum images without background color and without weight (b); Spectrum images with background color and with 300g weight(c); Spectrum images without background color and with 300g weight (d).

Through the spectrum images, changes are centralized in the center. In other words, target region is centralized near the origin. However, considering the spectrum spreading, circle with the origin as center, with 3 unit pixel as radius, is chosen. All the power spectrum values add together in this circle to decrease the effect of spectrum spreading. Weights are sequenced by size and the corresponding sum of power spectrum values can be obtained. Scatter diagram are displayed with this data.

Together with feature from TPS minimum bending energy, the sum of power spectrum value is combined to be sorted and further analyzed for their correlation with mass.

\section{Results and Discussion}

Results obtained from the two methods should be verified to test its validity and feasibility. According to the linear relation from the experiments, the theory value and the actual value of mass are compared to calculate their deviation ratio, where Deviation Ratio= (theory value - actual value)/ actual value. The smaller the value of the ratio, the higher the validity and feasibility are.

TPS minimum bending energy increases as the weights on belt increases, and black trend lines in Fig.5 (both left and right) can be drawn according to their corresponding bending energy represented by red points. Experimental conditions, such as the location of capturing pictures, lead to the difference between these two sets of data. In principle, two experimental results reflect the proportional linear relation between bending energy and weight in accordance. 

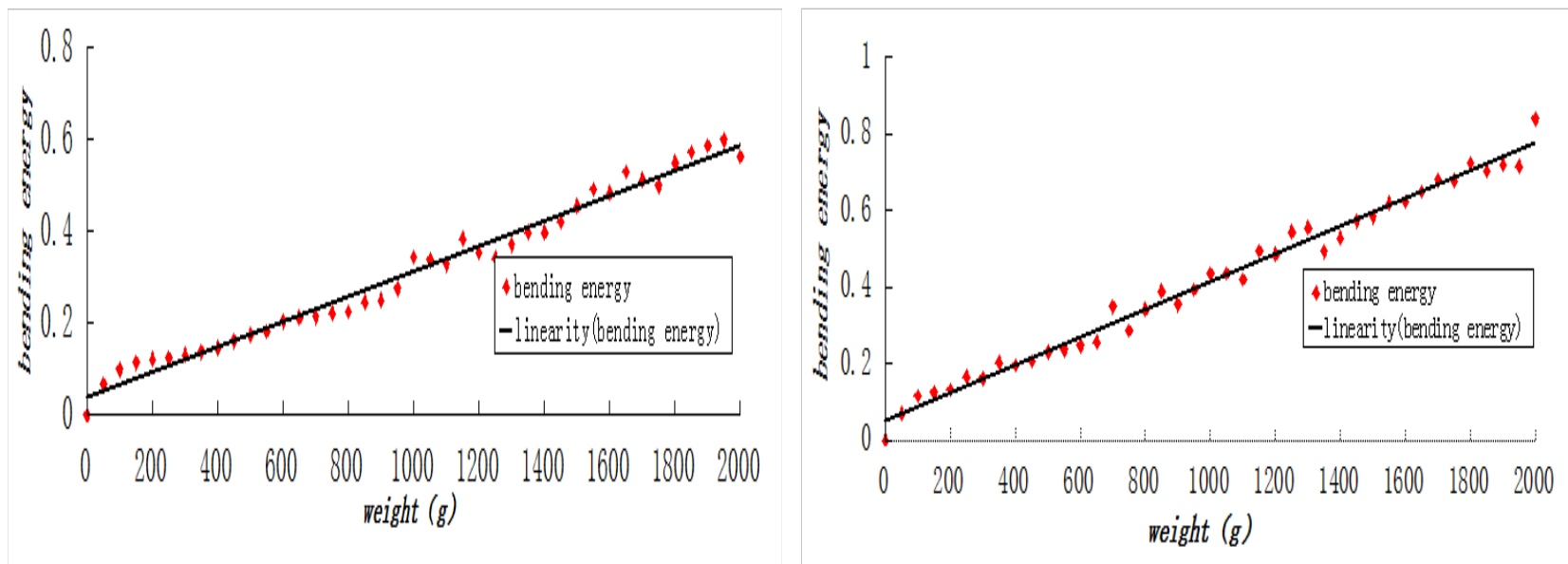

Fig.5 Experiment data distribution in spatial domain

The power spectrum value decreases as the weights on belt increases, shown with red points and black trend line in Fig.6 (both left and right). When forces being applied under the tensile belt, the distance of a grid dot changes, that corresponding to the change of single frequency of grid to multifrequency and causing the spread of power spectrum value to distribute in a wide region. Generally speaking, according to the difference of power spectrum value before and after deformation, the mass can be found out by its relationship to power spectrum.
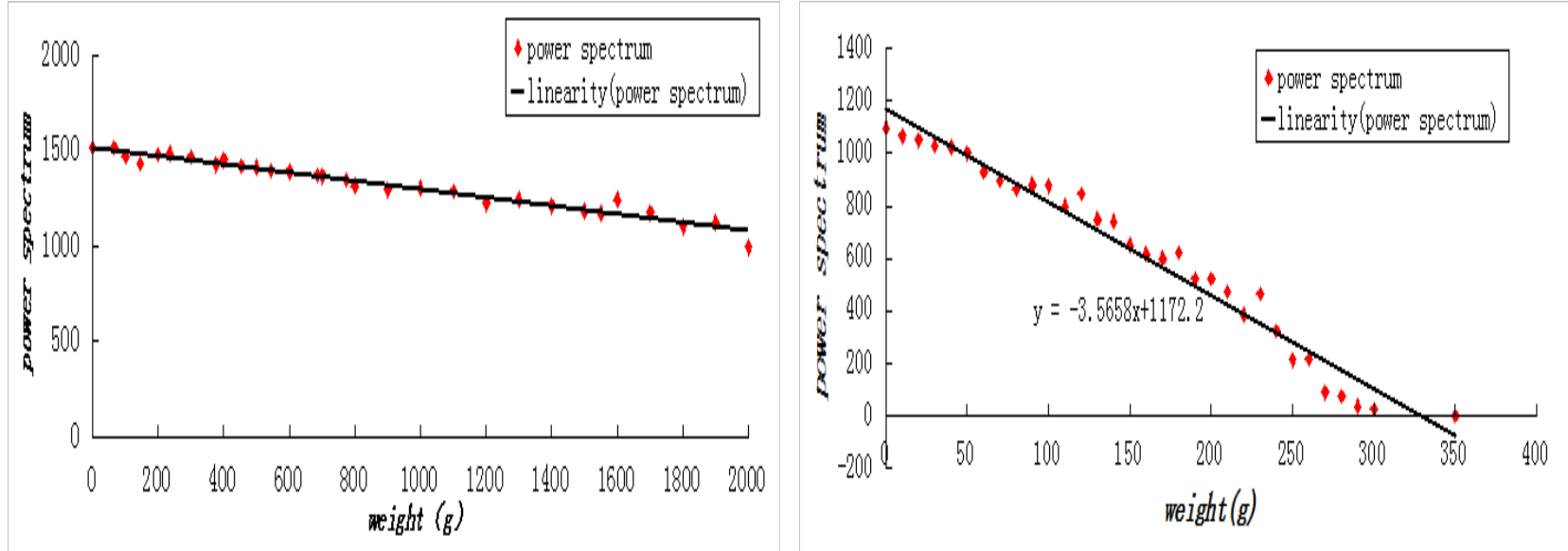

Fig.6 Experiment data distribution in frequency domain

Two sets of data in Fig.5 obtained from different mass $\left(\mathrm{m}_{1}\right)$ are combined and averaged, shown with blue points in Fig.7 (left). The black line is the trend line of data distribution. The points on the trend line can be chosen to obtain the mathematical expression: $y=0.000375 x$. According to its relation, data of bending energy in Fig.7 (left) is calculated to predict the mass $\left(\mathrm{m}_{2}\right)$. Then the error ratio between $m_{2}$ and $m_{1}$ can be calculated by formula (6) and yields $3.27 \%$, that means the deviation value is small in our experiment.
Deviation
Ratio
$(\mathrm{DR})=($
$\mathrm{m}_{1-}$
$\left.\mathrm{m}_{2}\right) /$
$\mathrm{m}_{2}$.

(6)

Similarly, data distribution in frequency domain is shown in Fig.7 (right) with the deviation ratio 0.0121429\% in average. Rectangle points in Fig.7 (right) represent the values of mass selected randomly except for the training data of mass (diamond points), which indicates the linear reciprocal relationship more generally. 

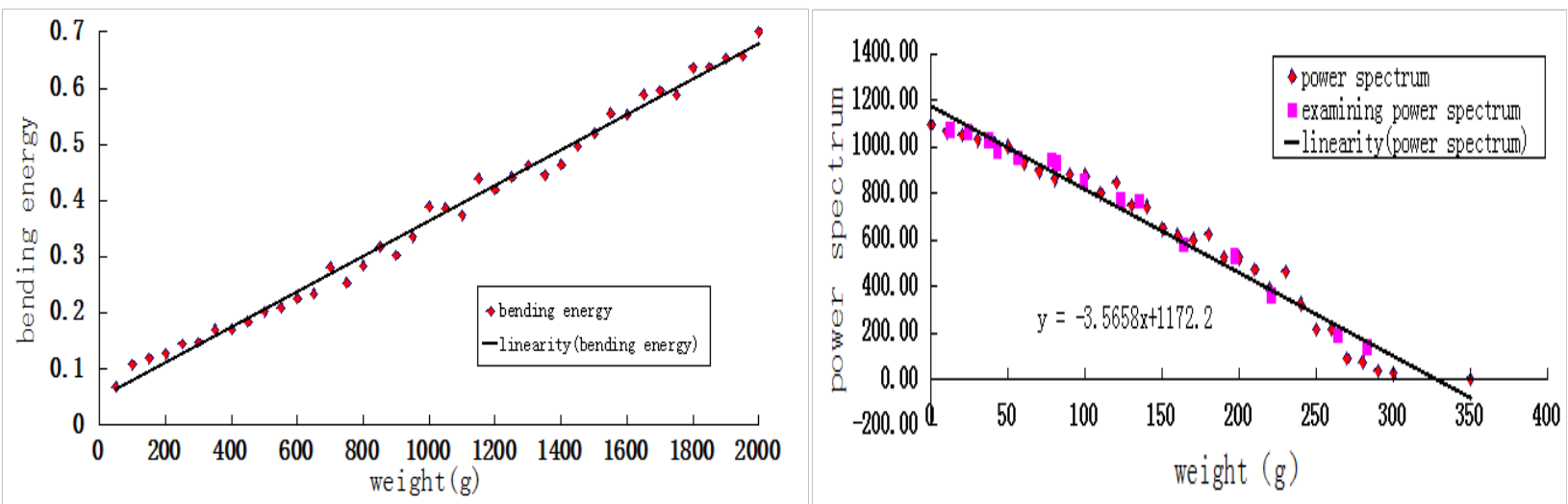

Fig.7 Experiment data distribution in spatial domain(left) and in frequency domain(right)

From the approximate curve we can notice that: In spatial domain, a linear proportional relationship between object mass and TPS minimum bending energy is shown for the material we selected, and the value of bending energy increases as the deformation becomes more remarkable. In frequency domain, it showed a linear reciprocal relationship between object mass and the sum of power spectrum value within the center of spectrum image: the power spectrum value decreases as the deformation becomes more remarkable. It is significant that TPS and FFT algorithm can measure the mass hanging on tensile belt accurately in both spatial and frequency domain. The algorithms can be easily extended to measure the mass of other objects. Furthermore, this method is now being applied to the measurement of the hardness of an object. If a fixed force pressing on two different materials, a soft one will have a greater deformation. On the contrary, smaller change of grids will be occurred on a hard object. By comparing the bending energy or power spectrum value of an unknown target with previous measured reference material, it is possible to quantify the hardness of an object we want to test. This is very useful for a Robert who with camera but no touching sensors wants to explore the hardness of an object it meeting.

\section{Conclusion}

This paper described the methods and experiments for object mass and hardness measurement based on image processing. Data with grid points is selected manually in spatial domain while automatically in frequency domain. The results show that there is a linear relationship between deformation degree and bending energy, as expected to the theory of prediction. Value of power spectrum within the center area also has linear relationship with deformation. To summary, object mass can be measured with spatial and frequency parameters in grid image sets, and it is expected that hardness can be also quantified by our proposed method.

\section{Acknowledgment}

This work was supported in part by two research support from the National Natural Science Foundation of China (No. 61262027 \& 45627390), and in part by a research foundation in Guangxi University for the students' experimental skills and scientific and technological innovation ability training project. The author Xuejun Zhang gratefully acknowledges financial support from China Scholarship Council (201308455018).

\section{References}

[1] E.A. Zerhouni, D.M. Parish, W.J. Rogers, A. Yang, and E.P. Shapiro, “Human heart: Tagging with MR imaging - a method for noninvasive assessment of myocardialmotion," Radiology, vol.169, no.1, pp.59-63, Oct. 1988.

[2] Xuejun Zhang, Satoshi Goshima, Xiangrong Zhou, Takeshi Hara, Masayuki Kanematsu, Hiroshi Fujita, Takahiro Furukawa, "Quantitative Staging the Hepatic Fibrosis: Computer-aided Diagnosis 
by Shape, Texture, Volume, and Elasticity Analysis", Proc. of Radiological Society of North America Scientific Assembly and Annual Meeting Program, Radiological Society of North America (RSNA), LL-INE1211, 2011.

[3] Franke, R.. "Smooth Interpolation of Scattered Data by Local Thin Plate Splines", Computer and Mathematics with Applications. Vol. 8, No. 4, pp.273-281,1982.

[4] L. Bookstein, "Principal warps: Thin-plate splines and the decomposition of deformations," IEEE Transactions on Pattern Analysis and Machine Intelligence, vol.11, no.6, pp.567-585, 1989.

[5] Heideman, M. T.; Johnson, D. H.; Burrus, C. S., "Gauss and the history of the fast Fourier transform". IEEE ASSP Magazine 1 (4): 14-21, 1984.. 